\title{
Simultaneous high PD-L1 and low VEGFR2 expression is associated with better overall survival in rectal cancer
}

\author{
Yun Ding ${ }^{1,2,3,4}$, Yuanyuan Fu ${ }^{1,2,3}$, Wei Wei ${ }^{1,2,3,5}$, Weibin Huang ${ }^{6}$, Zhuojun Zheng ${ }^{1,2,3}$, Dachuan Zhang ${ }^{1,2,3}$, \\ Jiajia $\mathrm{He}^{1,2,3,6}$, Xiao Zheng ${ }^{1,2,3}$, Qi Wang ${ }^{1,2,3}$, Yingting Liu ${ }^{1,2,3}$, Wendong Gu $^{1,2,3,4}$, Jingting Jiang ${ }^{1,2,3}$ \\ ${ }^{1}$ Department of Tumor Biological Treatment, The Third Affiliated Hospital of Soochow University, Changzhou, China; ${ }^{2}$ Jiangsu Engineering \\ Research Center for Tumor Immunotherapy, Changzhou, China; ${ }^{3}$ Institute of Cell Therapy, Soochow University, Changzhou, China; ${ }^{4}$ Department \\ of Radiation Oncology, The Third Affiliated Hospital of Soochow University, Changzhou, China; ${ }^{5}$ Department of Oncology, The Third Affiliated \\ Hospital of Soochow University, Changzhou, China; ${ }^{6}$ Department of Vascular Surgery, The Third Affiliated Hospital of Soochow University, \\ Changzhou, China \\ Contributions: (I) Conception and design: Y Ding, J Jiang; (II) Administrative support: J Jiang, X Zheng, Q Wang, Y Liu; (III) Provision of study \\ materials or patients: J Jiang, W Gu; (IV) Collection and assembly of data: Y Ding, W Wei, Y Fu, W Huang, D Zhang; (V) Data analysis and \\ interpretation: Z Zheng, W Wei, J He; (VI) Manuscript writing: All authors; (VII) Final approval of manuscript: All authors. \\ Correspondence to: Jingting Jiang. Department of Tumor Biological Treatment, The Third Affiliated Hospital of Soochow University, Juqian Road \\ No. 185, Changzhou 213003, China. Email: jiangjingting@suda.edu.cn.
}

Background: The aim of the present study was to analyze the association of programmed cell deathligand 1 (PD-L1) and vascular endothelial growth factor receptor 2 (VEGFR2) expression levels with clinicopathological characteristics and survival to provide a treatment strategy for rectal cancer.

Methods: Immunohistochemical staining of VEGFR2 and PD-L1 was carried out, and the association of PD-L1 and VEGFR2 expression levels with clinicopathological characteristics and survival were investigated in 77 pair-matched rectal cancer patients.

Results: PD-L1 and VEGFR2 expression levels in surgical tumor tissues were higher than those in paired adjacent normal tissues, respectively (both $\mathrm{P}<0.05$ ). The results of the 5 -year overall survival (OS) analysis showed that patients with low VEGFR2 expression $(66.7 \%$ vs. $43.5 \%, \mathrm{P}=0.042)$ and high tumor PD-L1 expression $(63.9 \%$ vs. $26.1 \%, \mathrm{P}=0.001)$ in tumor tissues demonstrated significantly better OS. Patients with high TNM stage had poorer OS [hazard ratio (HR): 2.093, 95\% confidence interval (CI): 1.027-4.087, $\mathrm{P}=0.030]$. Similar results of poorer OS could be seen in patients with low tumor PD-L1 expression (HR: 3.365, 95\% CI:1.747-6.481, $\mathrm{P}=0.005$ ), as well as patients with high tumor VEGFR2 expression (HR: 0.418, 95\% CI: $0.232-0.993, \mathrm{P}=0.048)$.

Conclusions: The results indicated that tumor PD-L1 and VEGFR2 expression levels were associated with OS, and the combination of tumor PD-L1 and VEGFR2 levels might be an independent prognostic factor in rectal cancer.

Keywords: Programmed cell death-ligand 1 (PD-L1); vascular endothelial growth factor receptor 2 (VEGFR2); overall survival (OS); rectal cancer

Submitted Oct 30, 2020. Accepted for publication Jan 14, 2021.

doi: $10.21037 /$ tcr-20-3106

View this article at: http://dx.doi.org/10.21037/tcr-20-3106 


\section{Introduction}

Colorectal cancer (CRC) is the third most common cancer and the fourth most common cause of cancer-related death in women and men worldwide (1). Therefore, it is important to determine novel treatment strategy in rectal cancer. Previous studies have found that low-dose apatinib treatment increases the infiltration of lymphocytes in tumors, and that a combination of low-dose apatinib and anti-programmed cell death-ligand 1 (PD-L1) treatment could significantly impact tumor growth and metastases, prolonging survival in mouse models (2). The expression of PD-L1 and vascular endothelial growth factor receptor 2 (VEGFR2) could be of great interest for the development of rectal cancer treatment.

Positive PD-L1 expression has been associated with significantly poor prognosis in various tumors; however, the prognostic significance of PD-L1 expression in rectal cancer still remains controversial (3-5). The evaluation of PD-L1 expression is also important in the application of immunotherapy and the prediction of therapeutic effects (4). Angiogenesis is a critical process during tumor progression and metastasis. VEGF/VEGFR2 signaling is another vital molecular pathway steering tumor angiogenesis, with multiple functions in tumor microenvironment (TME), including immunosuppression (6). Using a syngeneic lung cancer mouse model, Zhao et al. demonstrated that lowdose apatinib could alleviate hypoxia, increase $\mathrm{CD} 8^{+} \mathrm{T}$-cell infiltration, and reduce tumor-associated macrophage recruitment (2).

There is a need for specific prognostic biomarkers for rectal cancer patients in order to reach a more effective outcome prediction strategy. In the present study, we compared tumor PD-L1 and VEGFR2 expression levels in rectal cancer. We analyzed the relationship between the expression of PD-L1 and VEGFR2, as well their potential as biomarkers for prognostic prediction in rectal cancer.

We present the following article in accordance with the REMARK reporting checklist (available at http://dx.doi. org/10.21037/tcr-20-3106).

\section{Methods}

Commercially available rectal cancer tissue microarrays contented 90 rectal cancer tissue were purchased (HrecAde180Sur-03; Shanghai Outdo Biotechnology, Shanghai, China). Specimens were acquired from 90 rectal cancer patients who accepted surgery from July 2006 to August
2007. The follow-up period was 86 months. The followup endpoint was death. Tumors were staged according to the American Joint Committee on Cancer staging system. The following clinical pathological characteristics were collected: sex, age, TNM stage, pathological differentiation stage, and grade. The study was conducted in accordance with the Declaration of Helsinki (as revised in 2013). The present study was approved by the Ethics Committee of Soochow University. All enrolled patients provided signed informed consent.

The slides were dried, deparaffinized, rehydrated, antigen retrieval and endogenous peroxidases deactivation in order. The slides were incubated with VEGFR2 antibody (2479S, Cell Signaling Technology, USA) PD-L1 antibody (ab205921, Abcam, UK) at $4^{\circ} \mathrm{C}$ overnight. On the second day, anti-mouse/rabbit secondary antibody was added to the slices and incubated at room temperature for $30 \mathrm{~min}$ (Abcam, USA). A DAB substrate kit (DAB-0031, Fuzhou Maixin Biotech. Co., Ltd., Fuzhou, China) was then used for staining.

Tumor PD-L1 and VEGFR2 immunostaining was scored using the conventional Histoscore (H-score) calculation. The intensity of staining on a semiquantitative scale $(0-3+)$ is defined as follows: 0 , absent; $1+$, weak; $2+$, moderate; and $3+$, strong membrane staining. The possible range of $\mathrm{H}$-scores was from 0 to 300 .

\section{Statistical analysis}

Statistical analysis was conducted via GraphPad Prism 5.0 software (GraphPad Software, San Diego, California, USA) and SPSS version 21.0 (SPSS Inc., Chicago, IL, USA). Rank sum test, $\chi^{2}$-test, and Fisher's exact test were applied for group comparisons. The relationship between tumor expression levels of PD-L1, VEGFR2 and patient clinical parameters was evaluated using the Spearman's $\chi^{2}$ test. Influential factors of patient survival were adjusted in the Cox model. Kaplan-Meier analysis was applied to estimate the overall survival (OS) and to calculate the hazard ratios (HRs) of the collected parameters. All tests reported a 2 -sided $\mathrm{P}$ value with a significance level set at 0.05 .

\section{Results}

\section{Patient characteristics}

In total, 90 rectal cancer samples were collected. Of these, 6 cases were missing complete clinical data, and 7 lacked 
Table 1 Relationship between PD-L1 and VEGFR2 and patient characteristics $(\mathrm{n}=77)$

\begin{tabular}{|c|c|c|c|c|c|c|c|}
\hline Clinicopathological parameters & Cases $(\%)$ & \multicolumn{3}{|c|}{ PD-L1 } & \multicolumn{3}{|c|}{ VEGFR2 } \\
\hline Age (years) & & & & 0.411 & & & 0.546 \\
\hline$<65$ & $38(49)$ & 25 & 13 & & 24 & 14 & \\
\hline$\geq 65$ & $39(51)$ & 29 & 10 & & 22 & 17 & \\
\hline Female & $29(38)$ & 18 & 11 & & 16 & 13 & \\
\hline Male & $48(62)$ & 36 & 12 & & 30 & 18 & \\
\hline T stage & & & & 0.257 & & & 0.553 \\
\hline $\mathrm{T} 1-\mathrm{T} 2$ & $8(10)$ & 7 & 1 & & 4 & 4 & \\
\hline Negative & $42(55)$ & 32 & 10 & & 24 & 18 & \\
\hline Positive & $35(45)$ & 22 & 13 & & 22 & 13 & \\
\hline Distant metastasis & & & & 0.249 & & & 0.803 \\
\hline Negative & $74(96)$ & 51 & 23 & & 44 & 30 & \\
\hline Positive & $3(4)$ & 3 & 0 & & 2 & 1 & \\
\hline Pathological differentiation stage & & & & $0.015^{\star}$ & & & 0.659 \\
\hline$|-| \mid$ & $55(71)$ & 43 & 12 & & 32 & 23 & \\
\hline III & $22(29)$ & 11 & 11 & & 14 & 8 & \\
\hline \multicolumn{8}{|l|}{ Tumor PD-L1 } \\
\hline Low (\%) & 23 & & & & 13 & 10 & \\
\hline VEGFR2 & & & & 0.707 & & & \\
\hline High (\%) & 43 & 33 & 10 & & & & \\
\hline Low (\%) & 34 & 21 & 13 & & & & \\
\hline
\end{tabular}

*, significant at $\mathrm{P}<0.05$. $\chi^{2}$-test was used group comparisons. Fisher's exact test was used if a variant was $<5$. AJCC, American Joint Committee on Cancer; PD-L1, programmed cell death-ligand 1; VEGFR2, vascular endothelial growth factor receptor 2.

enough tumor tissue for this study, resulting in 77 valid cases for the present study. The follow-up period was 86 months. The median survival time was 74 months. The clinicopathological characteristics of these patients are presented in Table 1 . The majority of the patients were men $(62 \%)$, and the mean age at diagnosis was $59.4 \pm 12.5$ years (range, 31-90 years). Sixty-nine cases (90\%) were T3T4 stage. Thirty-five patients (45\%) had lymph node metastases, and 3 (4\%) had distant metastasis.

PD-L1 and VEGFR2 expression levels were examined using immunohistochemistry in both tumor tissues and paired adjacent normal tissues. Clinicopathological 

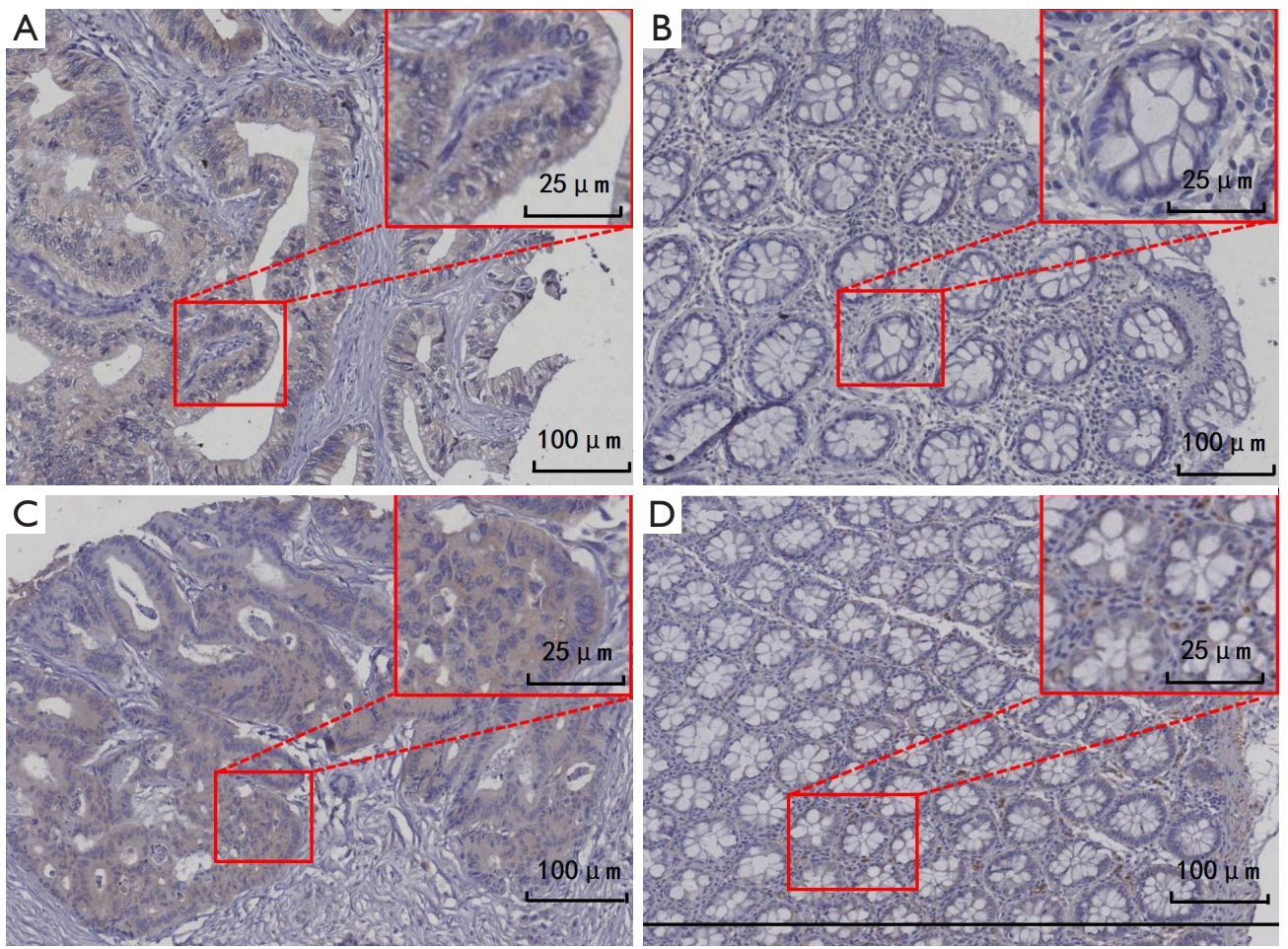

Figure 1 Programmed cell death-ligand 1 (PD-L1) and vascular endothelial growth factor receptor 2 (VEGFR2) immunohistochemistry in tumor tissue and adjust normal tissue for a patient with rectal cancer. (A) PD-L1 expression in tumor tissue. (B) PD-L1 expression in normal mucosa. (C) VEGFR2 expression in tumor tissue. (D) VEGFR2 expression in normal mucosa.

characteristics and the relationship with the expression levels of PD-L1 and VEGFR2 in surgical tumor tissues are presented in Table 1.

PD-L1 and VEGFR2 expression levels in tumor tissues and paired adjacent normal tissues

The expression levels of PD-L1 and VEGFR2 in surgical tumor tissues were higher than those in adjacent normal tissues, respectively (both $\mathrm{P}<0.05$ ) (Figure 1). Furthermore, we analyzed the relationship between clinicopathological parameters and PD-L1, as well as VEGFR2 expression, and found that a lower pathological differentiation stage was associated with higher PD-L1 expression $(\mathrm{P}=0.015)$.

\section{Tumor PD L1 expression and OS in patients}

We carried out a 5 -year OS analysis and found that patients with high tumor PD-L1 expression $(63.9 \%$ vs. $26.1 \%$, $\mathrm{P}=0.001)$ in tumor tissues had significantly better 5 -year OS (Table 2). Kaplan-Meier analysis also showed that patients with a high expression of tumor PD-L1 had better OS (log-rank $\mathrm{P}=0.001$ ) (Figure $2 A$ ). The findings demonstrate that high PD-L1 expression in tumor tissue indicate better clinical outcomes in rectal cancer.

Tumor VEGFR2 expression and OS in patients in the 5 -year OS analysis, patients with low VEGFR2 expression (66.7\% vs. $43.5 \%, \mathrm{P}=0.042)$ in tumor tissues exhibited significantly better 5 -year OS. Kaplan-Meier survival analysis also showed that patients with low tumor VEGFR2 level had better OS (log-rank $\mathrm{P}=0.042$ ) (Figure 2B). The findings demonstrate that low VEGFR2 expression could indicate better clinical outcomes in rectal cancer patients.

\section{Tumor PD-L1 and VEGFR2 association and OS differences in subgroups}

We conducted a Spearman's rank correlation test and found no significant relationship between the expression of $\mathrm{PD}$ L1 and VEGFR2 in rectal cancer $(\mathrm{P}=0.043$ and $\mathrm{P}=0.77$, respectively).

However, we observed survival differences between patients with different PD-L1 and VEGFR2 expression levels. Patients with high PD-L1 and low VEGFR2 levels 
Table 2 Clinicopathological parameters and 5-year overall survival

\begin{tabular}{|c|c|c|c|}
\hline $\begin{array}{l}\text { Clinicopathological } \\
\text { parameter }\end{array}$ & Cases & $\begin{array}{l}\text { 5-year overall } \\
\text { survival (\%) }\end{array}$ & $P$ value \\
\hline Age (years) & & & 0.304 \\
\hline$<65$ & 38 & 60.5 & \\
\hline$\geq 65$ & 39 & 51.3 & \\
\hline Sex & & & 0.734 \\
\hline Female & 29 & 55.2 & \\
\hline Male & 48 & 56.3 & \\
\hline T stage & & & 0.225 \\
\hline T1-T2 & 8 & 87.5 & \\
\hline T3-T4 & 69 & 52.2 & \\
\hline $\mathrm{pN}$ stage & & & $0.001^{*}$ \\
\hline Negative & 42 & 71.4 & \\
\hline Positive & 35 & 37.1 & \\
\hline Distant metastasis & & & $0.006^{\star}$ \\
\hline Negative & 74 & 58.1 & \\
\hline Positive & 3 & 0 & \\
\hline \multicolumn{2}{|c|}{ Pathological differentiation stage } & & 0.203 \\
\hline$I-I I$ & 55 & 60.0 & \\
\hline III & 22 & 45.5 & \\
\hline \multicolumn{2}{|c|}{ TNM stage (7th AJCC) } & & $0.016^{*}$ \\
\hline I-II & 42 & 71.4 & \\
\hline III-IV & 35 & 37.1 & \\
\hline Tumor PD-L1 & & & $0.001^{*}$ \\
\hline High & 54 & 63.9 & \\
\hline Low & 23 & 26.1 & \\
\hline Tumor VEGFR2 & & & $0.042^{*}$ \\
\hline High & 43 & 66.7 & \\
\hline Low & 34 & 43.5 & \\
\hline PD-L1/VEGFR2 & & & $0.001^{*}$ \\
\hline High/high & 33 & 57.6 & \\
\hline Low/low & 10 & 50 & \\
\hline High/low & 21 & 74.6 & \\
\hline Low/high & 13 & 7.7 & \\
\hline
\end{tabular}

*, significant at $P<0.05$. $P$ value was obtained from the log-rank test. Kaplan-Meier method was used for survival analysis. AJCC, American Joint Committee on Cancer; PD-L1, programmed cell death-ligand 1; VEGFR2, vascular endothelial growth factor receptor 2 . exhibited significantly better OS ( $\mathrm{P}=0.001)$ (Figure $2 C$ ). This suggests that high PD-L1 and low VEGFR2 expression levels could indicate a better prognostic factor in rectal cancer. T1-2 stage also exhibited significantly better OS than T3-4 stage $(\mathrm{P}=0.016)$ (Figure $2 D)$.

\section{Independent risk factors for rectal cancer patients}

In the univariate analysis, the OS rate of patients was associated with TNM stage, tumor PD-L1 expression and VEGFR2 expression $(\mathrm{P}<0.05)$. Patients with high TNM stage had poorer OS compared with that with low TNM stage [HR: 2.203, 95\% confidence interval (CI): 1.139 4.261, $\mathrm{P}=0.019]$. Similar risks for poorer OS could be seen in patients with low tumor PD-L1 expression (HR: 3.365, 95\% CI: $1.747-6.481, \mathrm{P}=0.005)$, and in those with a high tumor VEGFR2 expression (HR: 0.418, 95\% CI: 0.232 0.993, $\mathrm{P}=0.048$ ) (Table 3).

Subsequently, we a performed multivariate analysis, and the results were consistent with those of the univariate analysis. Patients with high TNM stage still had an increased risk for poorer OS compared with that with low TNM stage (HR:2.093, 95\% CI: 1.027-4.087, $\mathrm{P}=0.030$ ). Similar results could also be seen in patients with low tumor PD-L1 expression (HR: 4.566, 95\% CI: $2.237-$ 9.32), $\mathrm{P}=0.001)$, and in those with high tumor VEGFR2 expression (HR: 0.292, 95\% CI: 0.133-0.642, $\mathrm{P}=0.002$ ) (Table 3). These consistencies demonstrated that the combination of tumor PD-L1 and VEGFR2 levels could be an independent prognostic factor for rectal cancer patients.

\section{Discussion}

The association between PD-L1 and poor overall survival rate has been previously observed in melanoma, renal cancer, and lung cancer (7-9). However, other studies have found a correlation between tumor PD-L1 expression and improved survival outcomes in certain malignancies, such as melanomas, CRC, and non-triple-negative breast cancer $(3,10-12)$. Previous study showed that VEGFR2 expression in tumor was higher than that in normal cells, and VEGFR-2 shows an association with invasive adenocarcinoma and poor overall survival (13). It is reported that patients with negative PD-L1 expression in tumor had worse overall and recurrence-free survivals compared to patients with positive PD-L1 expression (3). A metaanalysis indicated that a high level of PD-L1 expression might be a biomarker for a poor prognosis in CRC 

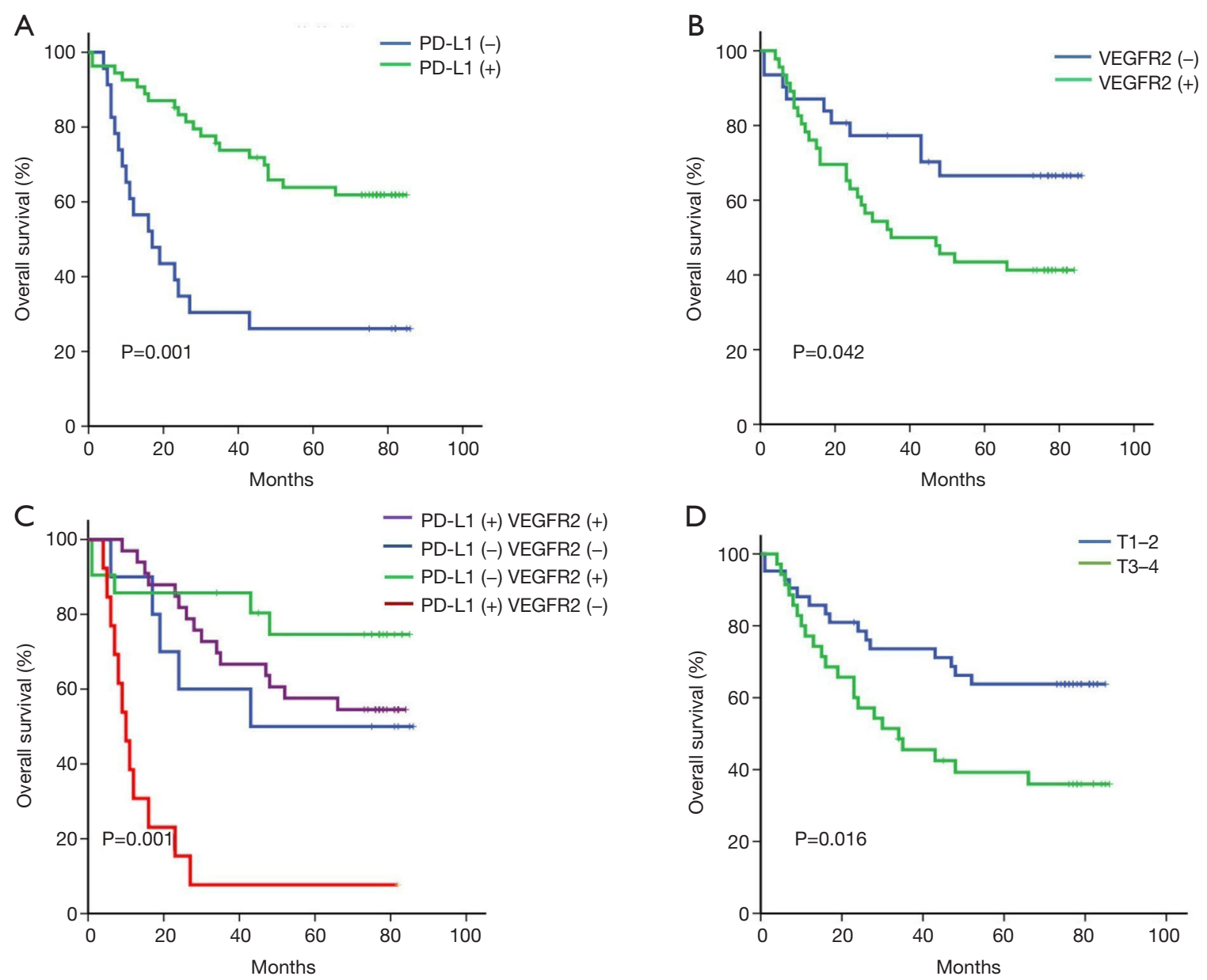

Figure 2 Kaplan-Meier curves of overall survival (OS) with tumor programmed cell death-ligand 1 (PD-L1) and vascular endothelial growth factor receptor 2 (VEGFR2) in rectal cancer patients. (A) Kaplan-Meier curves demonstrated that tumor PD-L1 expression is associated with OS in rectal cancer patients $(\mathrm{P}=0.001)$. (B) Kaplan-Meier curves demonstrated that tumor VEGFR2 expression is associated with OS in rectal cancer patients $(\mathrm{P}=0.042)$. (C) High tumor PD-L1 and low tumor VEGFR2 expressions within the tumor microenvironment are associated with improved $\mathrm{OS}$ in rectal cancer patients $(\mathrm{P}=0.001)$. (D) Kaplan-Meier curves demonstrated that tumor $\mathrm{T}$ stage is associated with OS in rectal cancer patients $(\mathrm{P}=0.016)$.

patients (4) Previous studies have indicated that patients with high PD-L1 have better OS and 5-year disease-free survival (DFS) (14). The advent of the PD-1/PD-L1inhibiting and anti-angiogenic therapy has improved the therapeutic effect of rectal cancer.

Previous studies have indicated that adjuvant sunitinib plus anti-PD-L1 improves OS compared with either drug alone in breast cancer models (15). Therefore, in the present study, we evaluated the correlation between the expression of PD-L1 and VEGFR2 with OS and found that patients with high PD-L1 and low VEGFR2 expression had better OS compared with that low PD-L1 and high VEGFR2 expression in rectal cancer. Angiogenesis is critical for tumor growth and metastasis. VEGF/VEGFR signaling plays an important role in tumor growth, progression, metastasis, angiogenesis, and in tumor microenvironment, including immunosuppression (6,16-20). Anti-angiogenic therapy targeting VEGF/VEGFR could transform the TME into an immunological favorable state, also described as a "hot" microenvironment. Antibodies targeting VEGF/ VEGFR are able to induce both anti-angiogenic and immune-supportive effects (21-23).

It has been reported that combination therapy with immunotherapy and/or anti-VEGF/VEGFR therapy 
Table 3 Univariate and multivariate analyses of clinicopathological parameters for 5-year overall survival

\begin{tabular}{|c|c|c|c|c|}
\hline Variable & \multicolumn{2}{|c|}{ Univariate analysis } & \multicolumn{2}{|c|}{ Multivariate analysis } \\
\hline Sex (male vs. female) & $1.041(0.535-2.023)$ & 0.906 & & \\
\hline Age ( $\geq 65$ vs. $<65$ years) & $0.962(0.505-1.834)$ & 0.907 & & \\
\hline T stage (T3-T4 vs. T1-T2) & $1.522(0.467-4.960)$ & 0.486 & & \\
\hline Pathological differentiation stage (III vs. I-II) & $1.457(0.730-2.906)$ & 0.285 & & \\
\hline TNM stage (III-IV vs. I-II) & $2.203(1.139-4.261)$ & $0.019^{*}$ & $2.093(1.072-4.087)$ & $0.030^{*}$ \\
\hline Tumor PD-L1 expression (low vs. high) & $3.365(1.747-6.481)$ & $0.005^{*}$ & $4.566(2.237-9.320)$ & $0.001^{*}$ \\
\hline Tumor VEGFR2 expression (low vs. high) & $0.418(0.232-0.993)$ & $0.048^{*}$ & $0.292(0.133-0.642)$ & $0.002^{*}$ \\
\hline
\end{tabular}

${ }^{*} \mathrm{P}<0.05$ was considered statistically significant. $\mathrm{Cl}$, confidence interval; HR, hazard ratio; PD-L1, programmed cell death-ligand 1; VEGFR2, vascular endothelial growth factor receptor 2.

produces favorable changes in the TME, and it has been suggested as a synergic treatment strategy in inhibiting proliferation and promoting the apoptosis of cancer cells (6,24-26). Patients with metastatic renal cell carcinoma who underwent anti-VEGF and anti-PD-L1 combination antibody therapy showed improved antigen-specific T-cell migration ability (26). This combined anti-VEGF and antiPD-L1 antibody therapy has been trialed in patients with recurrent glioblastoma renal cell carcinoma, CRC, and ovarian cancer (27). The results indicated that combination therapy consisting of immune checkpoint inhibitor and an anti-VEGF/VEGFR antibody could further benefit rectal cancer patients (28).

In the present study, we analyzed the relationship between clinicopathological parameters PD-L1 and VEGFR2, and the OS of 77 pair-matched rectal cancer tissues. Our result showed that PD-L1 and VEGFR2 positivity were significantly higher in cancer tissue than in normal tissue. Patients with a high level of PD-L1 expression and negative $\mathrm{pN}$ stage had better 5 -year OS. Similar to our findings, Chiang et al. showed that PD-L1 expression was associated with better DFS and 5-year OS in patients with locally advanced rectal cancer (14). Pyo et al. showed that PD-L1 expression was significantly correlated with favorable tumor behaviors and better OS (3). Patients with high tumor PD-L1 expression are most likely to benefit from immune checkpoint inhibitor treatment and improved survival outcomes in CRC $(3,29)$. These results suggest that $\mathrm{PD}-\mathrm{L} 1$ expression might be a prognostic factor for rectal cancer. High tumor PD-L1 expression induces immune microenvironment inhibition; therefore, it is reasonable to believe that checkpoint blockades might improve objective responses in PD-L1-high rectal cancer. This might help identify if those patients would benefit from immune checkpoint inhibitors.

Previous studies have shown that tumor PD-1/PDL1 expression is significantly associated with tumor stage and prognosis (21). In present study, we analyzed the relationship between PD-L1 and various patient clinicopathological characteristics, and found that high PD-L1 was significantly associated with low pathological differentiation stage. The results of the present study demonstrated that both PD-L1 and VEGFR2 expression levels were associated with the OS of rectal cancer patients. Patients with a high level of PD-L1 expression and/or with low VEGFR2 expression were found to have better OS. Kaplan-Meier analysis showed that, compared with other groups, patients with low VEGFR2 and high PDL1 expression had the best OS outcomes. Similar to our findings, previous studies have demonstrated that tumor PD-L1 expression is strongly associated with better OS outcomes among patients with non-triple-negative breast cancer, melanoma, and CRC (3,10-12).

Univariate and multivariate analyses showed that patients with high TNM stage exhibited an increased risk for poorer OS compared with patients with low TNM stage. Multivariate analysis models indicated that patients with high VEGFR2 and low PD-L1 expression levels had poorer OS than that with low VEGFR2 and high PD-L1 expression, indicating that tumor VEGFR2 and PD-L1 are 
prognostic factors for rectal cancer. A previous study proved that for colon cancer high PD-L1 expression was collected with less aggressive tumor behavior, leading to better OS (30). These results suggest that, for rectal cancer patients, upregulated tumor PD-L1 and downregulated tumor VEGFR2 indicate good clinical outcome. The findings of the present study indicate that combinational tumor PD-L1 and VEGFR2 expression levels may provide therapeutic strategies and predict outcomes for rectal cancer patients.

It has been reported that combined anti-PD-1/PDL1 and anti-VEGF/VEGFR2 antibodies demonstrate synergistic anti-tumor effects on some solid tumors (31). Anti-angiogenic therapy combined with PD-1/PD-L1 blockade was suggested as a potential new therapeutic approach for renal cell carcinoma patients (32). Shigeta et al. demonstrated that combined anti-PD-L1 and antiVEGFR2 therapy significantly inhibited primary tumor growth and improved survival in murine hepatocellular carcinoma models (33). Meder et al. indicated that dual anti-PD-L1/VEGF antibodies synergistically improved progression-free survival and OS in a mouse model of small cell lung cancer (30).

For rectal cancer, combining anti-VEGF/VEGFR2 antibody with immune checkpoint inhibitor treatment is potentially an efficient therapy strategy. In the future, a combination of checkpoint inhibitors and anti-angiogenic therapy should be established to improve the treatment efficacy of rectal cancer.

\section{Conclusions}

The findings of the present study highlight the impact of PD-L1 and VEGFR2 on rectal cancer by demonstrating their prognostic values in survival outcomes among rectal cancer patients. Based on tumor VEGFR2 and PD-L1 and expression levels in rectal cancer, novel prognostic and therapeutic strategies should be expected. Moreover, combined PD-L1 and VEGFR2 expression levels may be useful biomarkers to predict outcomes and establish the new treatment strategies based on the combination of immune checkpoint inhibitors and anti-angiogenesis antibodies to improve the treatment efficacy of rectal cancer.

\section{Acknowledgments}

Funding: The present study was supported by grants from the National Key R\&D Program (No. 2018YFC1313400), the National Key Technology R\&D Program (No. 2015BAI12B12), the National Natural Science Foundation of China (No. 81972869, 81902386, 31570877 , 31570908, 31729001), the Key R\&D Project of Science and Technology Department of Jiangsu Province (No. BE2018645), Changzhou Sci \& Tech Program (Grant No. CJ20190089, CJ20190101, CJ20190094, CJ20190088, CJ20180061, CJ20200113), and the Young Talent Development Plan of Changzhou Health Commission (No. CZQM2020021).

\section{Footnote}

Reporting Checklist: The authors have completed the REMARK reporting checklist. Available at http://dx.doi. org/10.21037/tcr-20-3106

Data Sharing Statement: Available at http://dx.doi. org/10.21037/tcr-20-3106

Conflicts of Interest: All authors have completed the ICMJE uniform disclosure form (available at http://dx.doi. org/10.21037/tcr-20-3106). The authors have no conflicts of interest to declare.

Ethical Statement: The authors are accountable for all aspects of the work in ensuring that questions related to the accuracy or integrity of any part of the work are appropriately investigated and resolved. The study was conducted in accordance with the Declaration of Helsinki (as revised in 2013). The present study was approved by the Ethics Committee of Soochow University. All enrolled patients provided signed informed consent.

Open Access Statement: This is an Open Access article distributed in accordance with the Creative Commons Attribution-NonCommercial-NoDerivs 4.0 International License (CC BY-NC-ND 4.0), which permits the noncommercial replication and distribution of the article with the strict proviso that no changes or edits are made and the original work is properly cited (including links to both the formal publication through the relevant DOI and the license). See: https://creativecommons.org/licenses/by-nc-nd/4.0/.

\section{References}

1. Bray F, Ferlay J, Soerjomataram I, et al. Global cancer statistics 2018: GLOBOCAN estimates of incidence and 
mortality worldwide for 36 cancers in 185 countries. CA Cancer J Clin 2018;68:394-424.

2. Zhao S, Ren S, Jiang T, et al. Low-dose apatinib optimizes tumor microenvironment and potentiates antitumor effect of PD-1/PD-L1 blockade in lung cancer. Cancer Immunol Res 2019;7:630-43.

3. Pyo JS, Ko SH, Ko YS, et al. Clinicopathological significance of PD-L1 expression in colorectal cancer: Impact of PD-L1 expression on pFOXO1 expression. Pathol Res Pract 2020;216:152764.

4. Li Y, He M, Zhou Y, et al. The Prognostic and Clinicopathological Roles of PD-L1 Expression in Colorectal Cancer: A Systematic Review and MetaAnalysis. Front Pharmacol 2019;10:139.

5. Pyo JS, Kang G, Kim JY. Prognostic role of PD-L1 in malignant solid tumors: a meta-analysis. Int J Biol Markers 2017;32:e68-74.

6. Tamura R, Tanaka T, Akasaki Y, et al. The role of vascular endothelial growth factor in the hypoxic and immunosuppressive tumor microenvironment: perspectives for therapeutic implications. Med Oncol 2019;37:2 .

7. Borghaei H, Paz-Ares L, Horn L, et al. Nivolumab versus Docetaxel in Advanced Nonsquamous Non-Small-Cell Lung Cancer. N Engl J Med 2015;373:1627-39.

8. Motzer RJ, Escudier B, McDermott DF, et al. Nivolumab versus Everolimus in Advanced Renal-Cell Carcinoma. N Engl J Med 2015;373:1803-13.

9. Larkin J, Chiarion-Sileni V, Gonzalez R, et al. Combined Nivolumab and Ipilimumab or Monotherapy in Untreated Melanoma. N Engl J Med 2015;373:23-34.

10. Baptista MZ, Sarian LO, Derchain SF, et al. Prognostic significance of PD-L1 and PD-L2 in breast cancer. Hum Pathol 2016;47:78-84.

11. Wu Z, Zhang L, Peng J, et al. Predictive and prognostic value of PDL1 protein expression in breast cancer patients in neoadjuvant setting. Cancer Biol Ther 2019;20:941-7.

12. Wang X, Liu Y. PD-L1 expression in tumor infiltrated lymphocytes predicts survival in triple-negative breast cancer. Pathol Res Pract 2020;216:152802.

13. Martins SF, Garcia EA, Luz MA, et al. Clinicopathological correlation and prognostic significance of VEGF-A, VEGF-C, VEGFR-2 and VEGFR-3 expression in colorectal cancer. Cancer Genomics Proteomics 2013;10:55-67.

14. Chiang SF, Huang CY, Ke TW, et al. Upregulation of tumor PD-L1 by neoadjuvant chemoradiotherapy (neoCRT) confers improved survival in patients with lymph node metastasis of locally advanced rectal cancers.
Cancer Immunol Immunother 2019;68:283-96.

15. Wu FTH, Xu P, Chow A, et al. Pre- and post-operative anti-PD-L1 plus anti-angiogenic therapies in mouse breast or renal cancer models of micro- or macro-metastatic disease. Br J Cancer 2019;120:196-206.

16. Gilbert MR, Dignam JJ, Armstrong TS, et al. A randomized trial of bevacizumab for newly diagnosed glioblastoma. N Engl J Med 2014;370:699-708.

17. Wen S, Shao G, Zheng J, et al. Apatinib regulates the cell proliferation and apoptosis of liver cancer by regulation of VEGFR2/STAT3 signaling. Pathol Res Pract 2019;215:816-21.

18. Ren X, Xie W, Wang Y, et al. VEGFR2-targeted fusion antibody improved NK cell-mediated immunosurveillance against K562 cells. Immunol Res 2016;64:1060-70.

19. Burkholder B, Huang RY, Burgess R, et al. Tumorinduced perturbations of cytokines and immune cell networks. Biochim Biophys Acta 2014;1845:182-201.

20. Gao Z, Shi M, Wang Y, et al. Apatinib enhanced antitumor activity of cisplatin on triple-negative breast cancer through inhibition of VEGFR-2. Pathol Res Pract 2019;215:152422.

21. Hato T, Zhu AX, Duda DG. Rationally combining antiVEGF therapy with checkpoint inhibitors in hepatocellular carcinoma. Immunotherapy 2016;8:299-313.

22. Tamura R, Tanaka T, Miyake K, et al. Bevacizumab for malignant gliomas: current indications, mechanisms of action and resistance, and markers of response. Brain Tumor Pathol 2017;34:62-77.

23. Tamura R, Tanaka T, Ohara K, et al. Persistent restoration to the immunosupportive tumor microenvironment in glioblastoma by bevacizumab. Cancer Sci 2019;110:499-508.

24. Liu XD, Hoang A, Zhou L, et al. Resistance to Antiangiogenic Therapy Is Associated with an Immunosuppressive Tumor Microenvironment in Metastatic Renal Cell Carcinoma. Cancer Immunol Res 2015;3:1017-29.

25. Hodi FS, Lawrence D, Lezcano C, et al. Bevacizumab plus ipilimumab in patients with metastatic melanoma. Cancer Immunol Res 2014;2:632-42.

26. Rexer H, Doehn C. First-line treatment for advanced renal cell carcinoma : A phase 3, open-label, randomized study of Atezolizumab (Anti-PD-L1-Antibody) in combination with Bevacizumab versus Sunitinib in patients with untreated advanced renal cell carcinoma ("IMmotion") AN 37/15 der AUO. Urologe A 2016;55:1242-3.

27. Omuro A, Vlahovic G, Lim M, et al. Nivolumab with 
or without ipilimumab in patients with recurrent glioblastoma: results from exploratory phase I cohorts of CheckMate 143. Neuro Oncol 2018;20:674-86.

28. Ott PA, Hodi FS, Buchbinder EI. Inhibition of Immune Checkpoints and Vascular Endothelial Growth Factor as Combination Therapy for Metastatic Melanoma: An Overview of Rationale, Preclinical Evidence, and Initial Clinical Data. Front Oncol 2015;5:202.

29. Chen TW, Huang KC, Chiang SF, et al. Prognostic relevance of programmed cell death-ligand 1 expression and CD8+ TILs in rectal cancer patients before and after neoadjuvant chemoradiotherapy. J Cancer Res Clin Oncol 2019;145:1043-53.

30. Wyss J, Dislich B, Koelzer VH, et al. Stromal PD-1/ PD-L1 Expression Predicts Outcome in Colon Cancer

Cite this article as: Ding Y, Fu Y, Wei W, Huang W, Zheng Z, Zhang D, He J, Zheng X, Wang Q, Liu Y, Gu W, Jiang J. Simultaneous high PD-L1 and low VEGFR2 expression is associated with better overall survival in rectal cancer. Transl Cancer Res 2021;10(1):499-508. doi: 10.21037/tcr-20-3106
Patients. Clin Colorectal Cancer 2019;18:e20-38.

31. Meder L, Schuldt P, Thelen M, et al. Combined VEGF and PD-L1 Blockade Displays Synergistic Treatment Effects in an Autochthonous Mouse Model of Small Cell Lung Cancer. Cancer Res 2018;78:4270-81.

32. Gunturi A, McDermott DF. Potential of new therapies like anti-PD1 in kidney cancer. Curr Treat Options Oncol 2014;15:137-46.

33. Shigeta K, Datta M, Hato T, et al. Dual PD-1 and VEGFR-2 blockade promotes vascular normalization and enhances anti-tumor immune responses in HCC. Hepatology 2020;71:1247-61.

(English Language Editor: R. Scott) 